\title{
Exhaled nitric oxide after lung transplantation: impact of the native lung
}

\author{
G.M. Verleden*,\#, L.J. Dupont*,\#, M. Delcroix*,\#, D. Van Raemdonck ${ }^{\boldsymbol{\top}, \#, ~ J . ~ V a n h a e c k e ~}{ }^{+, \#}$, T. Lerut, ${ }^{\boldsymbol{\Phi}, \#}$, \\ M. Demedts*,\#
}

Exhaled nitric oxide after lung transplantation: impact of the native lung. G.M. Verleden, L.J. Dupont, M. Delcroix, D. Van Raemdonck, J. Vanhaecke, T. Lerut, M. Demedts. C) ERS Journals Ltd 2003.

ABSTRACT: It has already been demonstrated that exhaled nitric oxide (eNO) is increased in lung transplant patients with chronic rejection, although it is not known whether the diseased native lung after single lung transplantation (SLTx) contributes to the increased eNO values. This study aimed to compare the eNO values in stable lung transplant patients (SLTx versus sequential (S)SLTx and heart (H)LTx) and in patients with established chronic rejection.

Altogether, 42 LTx patients (25 females, 13 SLTx, 18 SSLTx, 11 HLTx), with a mean follow-up of 1149 days and a mean age of 44.6 yrs at transplantation, were included. Twenty-six patients had no signs of chronic rejection (five SLTx and 21 SSLTx/HLTx). There was no difference in their eNO values (10.2 in SLTx versus 12.2 (parts per billion) ppb in SSLTx/HLTx). Sixteen patients (eight SLTx and eight SSLTx/HLTx) had a chronic rejection (eight bronchiolitis obliterans syndrome (BOS) potential stage, four BOS stage 1 , three BOS stage 2 and one BOS stage 3). Their eNOs were 18.1 (SLTx) and 17.0 (SSLTx/HLTx) ppb, respectively, which were significantly different to the stable LTx patients and showed a trend towards significance for SSLTx/HLTx. There was no significant difference in eNO between the patients with chronic rejection who underwent SLTx and those who underwent SSLTx/HLTx.

The diseased native lung after single lung transplantation probably does not contribute much to the exhaled nitric oxide values, either in stable lung transplant patients or in lung transplant patients with chronic rejection.

Eur Respir J 2003; 21: 429-432.
Depts of *Respiratory Diseases, "Cardiology, ${ }^{+}$Thoracic Surgery and ${ }^{\#}$ Lung Transplantation, University Hospital Gasthuisberg, Leuven, Belgium.

Correspondence: G.M. Verleden

Dept Respiratory Disease and Lung

Transplantation Unit

University Hospital Gasthuisberg

49 Herestraat

B-3000 Leuven

Belgium

Fax: 3216346803

E-mail: geert.verleden@uz.kuleuven. ac.be

Keywords: Exhaled nitric oxide lung transplantation obliterative bronchiolitis

Received: March 212002

Accepted after revision: October 18 2002
Although the results of lung transplantation (LTx) have improved dramatically in recent years, bronchiolitis obliterans syndrome (BOS), the clinical correlate or obliterative bronchiolitis $(\mathrm{OB})$, the pathological correlate of chronic rejection, still hampers the long-term survival after this procedure and remains the leading cause of morbidity and late mortality [1]. Several risk factors for the development of OB/BOS have now been identified, of which late and recurrent acute rejection are the most widely accepted up to now [2]. Other risk factors include lymphocytic bronchitis/bronchiolitis, human leukocyte antigen mismatches at the A locus, total human leukocyte antigen mismatches and cytomegalovirus pneumonitis [2]. Tackling these risk factors may, in turn, decline the prevalence of chronic rejection [3], although this remains to be established.

Since it has been demonstrated that survival is much better in lower BOS stages [4], it has become clear that early detection and treatment of chronic rejection is probably one of the most important factors that may improve the final outcome [5]. In recent years, different markers have been identified that may help early diagnosis of $\mathrm{OB} / \mathrm{BOS}$, such as high-resolution computed tomography [6], indexes of ventilation distribution (although the latter technique was only useful after sequential single lung transplantation (SSLTx) and heart/lung transplant (HLTx) [7]) and the early presence of aspecific bronchial hyperresponsiveness, which was, again, only investigated in recipients of an SSLTx [8].

It has been recently demonstrated that patients with chronic rejection have an increased exhaled nitric oxide (eNO) compared to stable LTx patients and healthy, nonsmoking volunteers [9-11]. Since it has also been demonstrated, in a further prospective study, that the increase in eNO levels occurs at a mean of 8.6 months before BOS stage 1 can be diagnosed, this noninvasive test might be very useful for early diagnosis of chronic rejection after lung transplantation [12]. Currently, however, it is not known whether the native lung after single lung transplantation (SLTx) contributes to the eNO levels measured after transplantation.

Therefore, the aim of the present study was to investigate the possible contribution of the native lung to eNO measurements in the stable condition and during chronic rejection.

\section{Patients and methods}

The University Hospital Gasthuisberg Ethics Committee (Leuven, Belgium) approved the study and informed consent was obtained from each patient. Altogether, 42 consecutive lung transplant patients were recruited in this cross-sectional study. They were divided according to their rejection status, based on the published BOS criteria [13], that were recently revised to include a potential BOS (BOS O-p) stage, which already denotes a decline in forced expiratory volume in one second (FEV1) or forced expiratory flow at $25-75 \%$ of forced vital capacity (FEF25-75\%), although the possible evolution to established chronic rejection in this group is not yet clear [5].

The mean age of the 42 patients was 44.6 yrs (SD 12.5, 
range 15-62) and there were 25 females. Thirteen patients received an SLTx (eight for chronic obstructive pulmonary disease (COPD)/emphysema and five for pulmonary fibrosis, including three for cryptogenic pulmonary fibrosis, one for histiocytosis $\mathrm{X}$ and one for lung fibrosis associated with scleroderma). Eighteen patients underwent SSLTx (11 for COPD/emphysema, including four for $\alpha_{1}$-antitrypsine deficiency emphysema, six for cystic fibrosis and one for cryptogenic pulmonary fibrosis). Eleven patients underwent HLTx (five for primary pulmonary hypertension, four for Eisenmenger's syndrome, one for secondary pulmonary hypertension due to cirrhosis and one for COPD/emphysema). They had a follow-up of 1,149 days (SD 935, range 117-4382) at the time of the study. Twenty-six patients had absolutely no evidence of chronic rejection (five SLTx, 14 SSLTx and seven HLTx). Of the remaining 16 patients (eight SLTx and eight SSLTx/HLTx), eight were in BOS O-p (three SLTx, two SSLTx and three HLTx), four in BOS one (three SLTx and one SSLTx), three in BOS two (two SLTx and one SSLTx) and one in BOS three (HLTx).

Twenty healthy subjects ( 12 females), recruited from among the staff of the University Hospital Gasthuisberg were used as a control group. Their mean age was 42 yrs (SD 12). All were nonsmokers and none of them had any respiratory complaints. Neither the control group nor the LTx patients had a history of upper respiratory tract infection for $\geqslant 4$ weeks prior to the measurements of eNO. Neither patients nor control subjects consumed any alcoholic or caffeinated beverages in the $8 \mathrm{~h}$ prior to the eNO measurements. All LTx patients were treated with cyclosporine A and oral corticosteroids in combination with azathioprine when the leukocyte count was $>4,000 \mathrm{~mm}^{-3}$

Exhaled NO was measured according to earlier publications from the authors' group [14, 15] and in line with European Respiratory Society guidelines [16], using a chemiluminescence analyser (Ecophysics, Dürnten, Switzerland), with a sampling rate of $0.7 \mathrm{~L} \cdot \mathrm{s}^{-1}$ and a response time of $1 \mathrm{~s}$. Three reproducible recordings were made at 2-min intervals and the highest of the three readings was used for analysis. All eNO levels are reported in parts per billion (ppb). All measurements were performed between 10:00 and 11:00 h.

The measurements of eNO were compared by means of the multiple comparisons analysis of variance (ANOVA) test (with a Bonferroni post hoc correction) and a $2 * 2$ ANOVA. Correlation between BOS stage and eNO was evaluated using the Spearman Rank correlation coefficient, taking patients in BOS stages 2 and 3 together as one group. Data are given as mean \pm SD. A significance level was set at $\mathrm{p}<0.05$.

A post hoc power analysis revealed a power of $75 \%$ to detect no significant difference between the stable LTx patients and the healthy volunteers on the one hand and a power of $<10 \%$ to detect no significant difference between the BOS patients after SLTx and after SSLTx/HLTx on the other hand. The power to detect a significant difference $(\mathrm{p}<0.05$, eNO difference of $5 \mathrm{ppb}$ ) between stable and BOS patients after SLTx and after SS/HLTx was $45 \%$ and $60 \%$, respectively.

\section{Results}

The eNO values in the 26 patients in a stable condition who underwent either SLTx or SSLTx/HLTx were 10.2 \pm 2.3 and $12.2 \pm 3.5 \mathrm{ppb}$, respectively $(\mathrm{p}=1 ; 95 \%$ confidence interval (CI) -8.3-4.3). There was no significant difference in eNO between these 26 stable LTx patients and the 20 healthy controls (11.8 \pm 3.4 versus $10.0 \pm 2.0 \mathrm{ppb}, \mathrm{p}=1$; 95\% CI $-6.3-4.3$ ).

The eNO value of the eight SLTx patients with chronic rejection was $18.1 \pm 7.1 \mathrm{ppb}$, which was significantly different from the eNO value of the five stable SLTx patients
$(10.2 \pm 2.3, \mathrm{p}=0.022 ; 95 \% \mathrm{CI}-11.2$ to -0.7$)$ but was the same as the eNO value of the eight SSLTx/HLTx patients with chronic rejection $(17.0 \pm 7.5, \mathrm{p}=1 ; 95 \% \mathrm{CI}-5.2-7.4)$. However, the eNO value of this latter group of patients showed a trend towards a significant difference from the eNO value of the 21 SSLTx/ HLTx patients that remained stable $(17.0 \pm 7.5$ and $12.2 \pm 3.5 \mathrm{ppb}$, respectively, $\mathrm{p}=0.071 ; 95 \% \mathrm{CI}-0.3-9.9)$. There was no significant difference in eNO between the patients with chronic rejection who underwent SLTx versus those who underwent SSLTx/ HLTx (18.1 \pm 7.1 versus $17.0 \pm 7.5, \mathrm{p}=1$; 95\% CI -5.2-7.4).

Using $2 * 2$ ANOVA, the only significant interaction that was found was between the stable patients and the BOS patients in both groups $(\mathrm{p}=0.001)$. There was no significant interaction between the two stable groups of patients $(\mathrm{p}=0.814)$ nor between the two BOS patient groups $(\mathrm{p}=0.398)$.

For all patients $(n=42)$, there was a significant correlation between BOS stage and eNO ( $\mathrm{rho}=0.557, \mathrm{p}<0.0001)$ (data not shown). Taking an eNO value of $\geqslant 15 \mathrm{ppb}$ as the cut-off value, the sensitivity and specificity of eNO to detect BOS was $100 \%$ and $45 \%$, respectively. All eNO values are summarised in figure 1 .

\section{Discussion}

The present study has confirmed that patients with chronic rejection after LTx have an increased eNO value compared to stable LTx patients and healthy controls. Furthermore, there seems to be no difference in eNO between either SLTx patients or SSLTx/HLTx patients. The fact that the difference in eNO between stable SSLTx/HLTx patients and BOS patients after the same procedure only showed a trend towards a significant difference, may be explained by the fact that the two groups are very different with regard to the number of patients (21 versus eight).

Although the eNO values show an overlap between the different groups (fig. 1), the sensitivity and the specificity of an eNO value of $\geqslant 15 \mathrm{ppb}$ to detect BOS is reasonable $(100 \%$ and $45 \%$, respectively). Since the numbers of patients per group are rather small, the power of the study is not particularly high, but is still acceptable $(45-70 \%)$, except for the

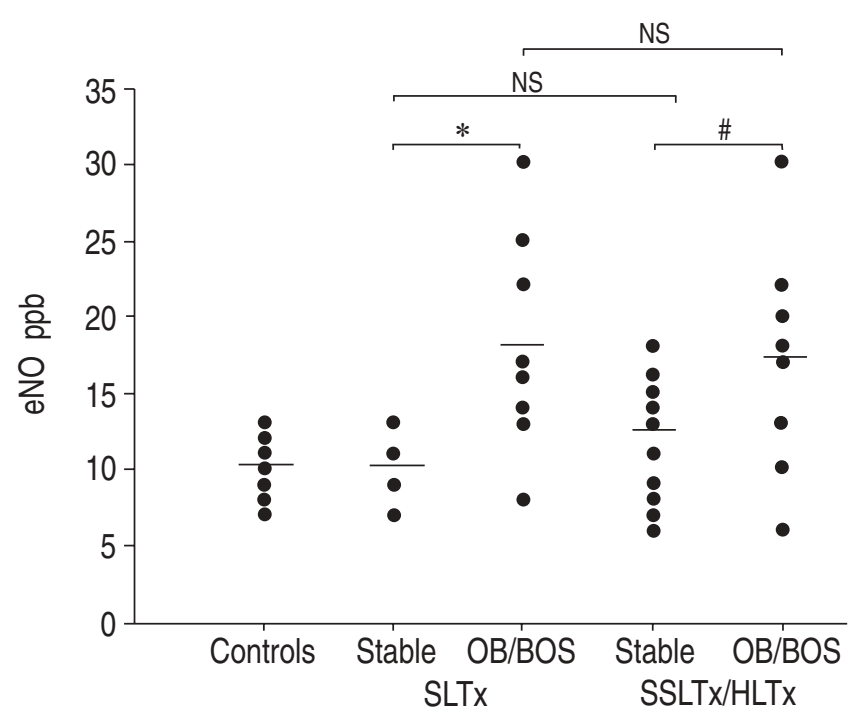

Fig. 1.- Individual values of exhaled nitric oxide (eNO) in different patient groups. ppb: parts per billion; OB: obliterative bronchiolitis; BOS: bronchiolitis obliterans syndrome; SLTx: single lung transplantation; SSLTx: sequential SLTx; HLTx: heart/lung transplantation. $\ldots$ : mean value. NS: nonsignificant. ${ }^{*}: \mathrm{p}<0.05{ }^{*}: \mathrm{p}=0.071$. 
comparison between the BOS patients in the two groups, which might be a drawback in the interpretation of the results. However, the present data still confirms earlier published data showing that eNO is increased in patients with chronic rejection after LTx [9-11], and, at the least, argues against a difference in eNO between SL and SSLTx/HLTx in the stable and in the BOS condition.

The authors have also recently demonstrated via a prospective study that eNO can indeed be used in the early diagnosis of chronic rejection [12]. Furthermore, the authors have demonstrated, for the first time, that there is no significant difference in eNO values between recipients of a SLTx compared to recipients of a SSLTx/HLTx, either in a stable condition or during chronic rejection.

The measurement of eNO could be of the utmost importance in the early detection of chronic rejection and/or lymphocytic bronchitis/bronchiolitis [9-11], as it has been demonstrated that eNO increased during the 8 months before BOS 1 can be diagnosed based on the classical FEV1 criteria [12]. However, because other markers of early detection have only been used in recipients of SSLTx/HLTx [7, 8] or are difficult to interpret in SLTx recipients (for instance, the FEF25-75\%), it still had to be confirmed that eNO values do not depend upon the nature of the transplantation procedure and, hence, that the native lung after SLTx has no major impact on the measurement of eNO. This is of particular interest, since it has previously been demonstrated that the eNO value in COPD patients is increased [17] and that eNO values correlate negatively with FEV1, with the lower FEV1 values corresponding to higher eNO values [18]. Moreover, an association has been found between eNO values and bronchial inflammation in COPD patients, as assessed by using induced sputum neutrophilia and interleukin-8 [19]. Also, in fibrosing lung diseases, such as these in association with scleroderma and cryptogenic fibrosing alveolitis, it has been found that eNO values are increased and there was even a correlation between the eNO values and the lymphocyte count in the bronchoalveolar lavage fluid in patients with lung fibrosis associated with scleroderma [20].

Despite these findings, the present study could not demonstrate any significant difference between the eNO measurements in recipients of a SLTx compared to recipients of SSLTx/HLTx, which suggests that the impact of the native lung is negligible when interpreting the eNO values after LTx, as well in a stable situation as during chronic rejection. Another possible drawback could be a difference in BOS grading between the two LTx groups, which might explain why no difference in eNO values could be demonstrated. It is well known that eNO is especially increased in lower BOS stages and even in patients with lymphocytic bronchitis/ bronchiolitis, which may be compatible with the BOS O-p stage and seems to be reversible upon treatment with inhaled steroids [11], which none of the patients in the present study were using. However, when comparing the BOS stages in the two patient groups, six of the eight patients with chronic rejection after SLTx were in BOS O-p and BOS stage 1 and, in the other patient group, six of the eight with chronic rejection were also in the same BOS stages.

Although eNO is also increased during infectious episodes, this is irrelevant for the current study, due to the exclusion of every patient with an infection during the previous 4 weeks and with a clinical infection at the time of the eNO measurements. Because of the rigid use of the BOS criteria [13, 14], other causes of a decline in FEV1 can also be excluded.

In various disease conditions, such as asthma [14, 15], bronchiectasis [21], COPD [19] and pulmonary fibrosis [20], a correlation has been found between indexes of bronchial inflammation and eNO. In stable lung transplant recipients, eNO positively correlates with airway neutrophilia and bronchial epithelial inducible nitric oxide synthase (iNOS) [22], whereas BOS is characterised by increased epithelial iNOS [23]. Since eNO correlates with inflammatory airways parameters and since it is already increased in very early (and potentially reversible) stages of chronic rejection [11], which is again confirmed in the present study, this measurement seems very important as an early marker of chronic airways inflammation and, hence, possible early stages of chronic rejection. A study has recently been published that stated that upregulation of iNOS in fibroblasts parallels the onset and progression of fibrosis in an experimental model of post-transplant obliterative airway disease, characterised by an infiltration of polymorphonuclear cells and lymphocytes [24].

Since the authors have now demonstrated that eNO measurements can be used in all lung transplant patients, independent of the transplantation procedure itself, this test may become very important in the follow-up of lung transplant recipients, since an increased eNO (in the absence of other conditions that lead to an increased eNO, such as a recent infection) may point to very early airways inflammation, which might be the hallmark of an ongoing chronic rejection. Moreover, by using this test in the routine follow-up of lung transplant recipients, the onset of chronic airways inflammation (rejection) in a still reversible stage might be diagnosed [12]. However, there is still a potential problem since the eNO test is not particularly specific $(45 \%)$, although very sensitive $(100 \%)$ for diagnosis of chronic rejection in lung transplant patients, especially when only one measurement of eNO $\geqslant 15 \mathrm{ppb}$ is taken into account.

In conclusion, it has been demonstrated that the measurement of exhaled nitric oxide is not influenced by the presence of the native lung after single lung transplantation, which makes this test useful for the possible early detection of chronic rejection in all lung transplant recipients, independent of the operative procedure.

\section{References}

1. Hosenpud JD, Bennett LE, Keck BM, Moucek M, Novick RJ. The registry of the international society for heart and lung transplantation: seventeenth official report - 2000. J Heart Lung Transplant 2000; 19: 909-931.

2. Kroshus TJ, Kshettry VR, Savik K, Ranjit J, Herz MI, Bolman RM. Risk factors for the development of bronchiolitis obliterans syndrome after lung transplantation. $J$ Thorac Cardiovasc Surg 1997; 114: 195-202.

3. Verleden GM. Bronchiolitis obliterans syndrome after lung transplantation: medical treatment. Monaldi Arch Chest Dis 2000; 55: 140-145.

4. Reichenspurner H, Girgis RE, Robbins RC, et al. Bronchiolitis obliterans after lung and heart lung transplantation. Ann Thorac Surg 1995; 60: 1845-1853.

5. Verleden GM. Chronic allograft rejection (obliterative bronchiolitis). Sem Respir Crit Care Med 2001; 22: 551-557.

6. Ikonen T, Kivisaari L, Taskinen E, Piilonen A, Harjula AL. High-resolution CT in long-term follow-up after lung transplantation. Chest 1997; 111: 370-376.

7. Estenne M, Van Muylem A, Knoop C, Antoine M. Detection of obliterative bronchioitis after lung transplantation by indexes of ventilation distribution. Am J Respir Crit Care Med 2000; 162: 1047-1051.

8. Stanbrook MB, Kesten S. Bronchial hyperreactivity after lung transplantation predicts early bronchiolitis obliterans. Am J Respir Crit Care Med 1999; 160: 2034-2039.

9. Verleden GM, Dupont LJ, Lamont J, et al. Is there a role for measuring exhaled nitric oxide in lung transplant recipients with chronic rejection? J Heart Lung Transplant 1998; 17: 231-232.

10. Fisher AJ, Gabbay E, Small T, Doig S, Dark JH, Corris PA. 
Cross sectional study of exhaled nitric oxide levels following lung transplantation. Thorax 1998; 53: 454-458.

11. De Soyza A, Fisher AJ, Small T, Corris PA. Inhaled corticosteroids and the treatment of lymphocytic bronchiolitis following lung transplantation. Am J Respir Crit Care Med 2001; 164: 1209-1212.

12. Verleden GM, Buyse B, Delcroix M, Dupont L, Van Raemdonck D, Vanhaecke J. Exhaled nitric oxide (eNO) after lung transplantation (LTx): a prospective study. J Heart Lung Transplant 2001; 20: 208.

13. Cooper JD, Billingham $\mathrm{M}$, Egan $\mathrm{T}$, et al. A working formulation for the standardization of nomenclature for clinical staging of chronic dysfunction in lung allografts. J Heart Lung Transplant 1993; 12: 713-726.

14. Dupont LJ, Rochette F, Demedts MG, Verleden GM. Exhaled nitric oxide correlates with airway hyperresponsiveness in steroid-naive patients with mild asthma. Am J Respir Crit Care Med 1998; 157: 894-898.

15. Verleden GM, Dupont LJ, Verpeut AC, Demedts MG. The effect of cigarette smoking on exhaled nitric oxide in mild steroid-naive asthmatics. Chest 1999; 116: 59-64.

16. Kharitonov S, Alving K, Barnes PJ. Exhaled and nasal nitric oxide measurements: recommendations. Eur Respir J 1997; 10: $1683-1693$.

17. Montuschi P, Kharitonov SA, Barnes PJ. Exhaled carbon monoxide and nitric oxide in COPD. Chest 2001; 120: 496501.
18. Ansarin K, Chatkin JM, Ferreira IM, Gutierrez CA, Zamel N, Chapman KR. Exhaled nitric oxide in chronic obstructive pulmonary disease: relationship to pulmonary function. Eur Respir J 2001; 17: 934-938.

19. Silkoff PE, Martin D, Pak J, Westcott JY, Martin RJ. Exhaled nitric oxide correlated with induced sputum findings in COPD. Chest 2001; 119: 1049-1055.

20. Paredi P, Kharitonov SA, Loukides S, Pantelidis P, du Bois RM, Barnes PJ. Exhaled nitric oxide is increased in active fibrosing alveolitis. Chest 1999; 115: 1352-1356.

21. Kharitonov SA, Wells AU, $\mathrm{O}^{\prime}$ Connor BJ, et al. Elevated levels of exhaled nitric oxide in bronchiectasis. Am J Respir Crit Care Med 1995; 151: 1889-1893.

22. Gabbay E, Walters H, Orsida B, et al. In stable lung transplant recipients, exhaled nitric oxide levels positively correlate with airway neutrophilia and bronchial epithelial iNOS. Am J Respir Crit Care Med 1999; 160: 2093-2099.

23. Gabbay E, Walters H, Orsida B, et al. Post-lung transplant bronchiolitis obliterans syndrome (BOS) is characterized by increased exhaled nitric oxide levels and epithelial inducible nitric oxide synthase. Am J Respir Crit Care Med 2000; 162 : 2182-2187.

24. Romanska HM, Ikonen TS, Bishop AE, Morris RE, Polak JM. Up-regulation of inducible nitric oxide synthase in fibroblasts parallels the onset and progression of fibrosis in an experimental model of post-transplant obliterative airway disease. J Pathol 2000; 191: 71-77. 